\title{
CASE FOR DIAGNOSIS
}

\section{A female with cough, dyspnoea and chest pain}

\author{
S. Suma*, P. Polat*, M. Görgüner ${ }^{\#}$
}

\section{Case history}

A 42-yr-old female presented with complaints of cough, dyspnoea and chest pain for 2 months. She was living in the eastern part of Turkey. As a child, she lived in the countryside, and her family earned their living by raising cattle. She has lived in the city for 10 yrs and did not have any animals in her house. There was neither sputum nor haemoptysis. She had lost weight, $\sim 3 \mathrm{~kg}$ in 4 months. Physical and biochemical examinations showed no apparent pathologies.

A chest roentgenogram is shown in figure 1. A computed tomography $(\mathrm{CT})$ section from the chest is shown in figure 2.

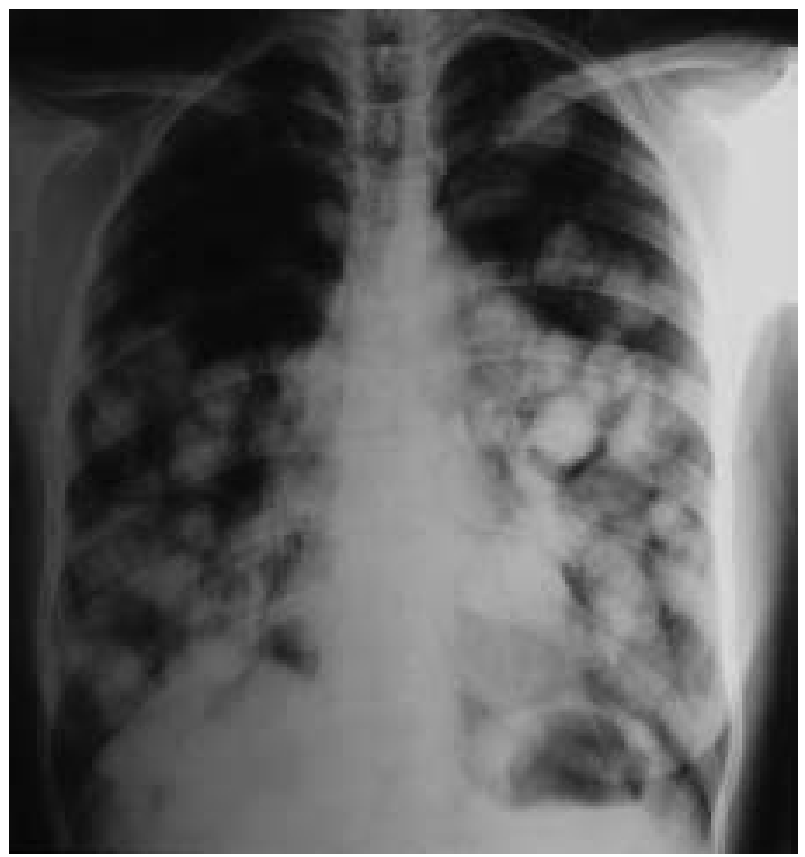

Fig. 1. - Posteroanterior chest radiography.
Ultrasound examination of pleura-based lung lesions revealed anechoic cystic lesions. A CT-guided fine-needle aspiration was performed and $15 \mathrm{~mL}$ clear-white fluid was evacuated; the findings are shown in figure 3 .

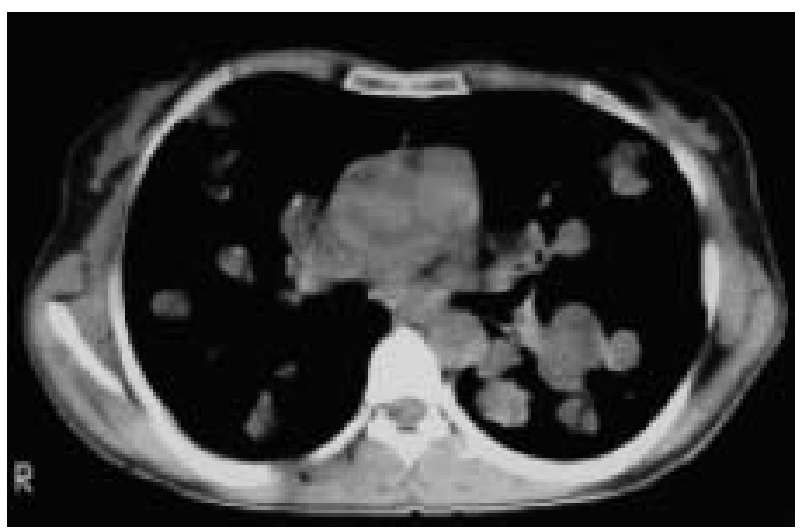

Fig. 2.-Axial computed tomography sections through the chest.

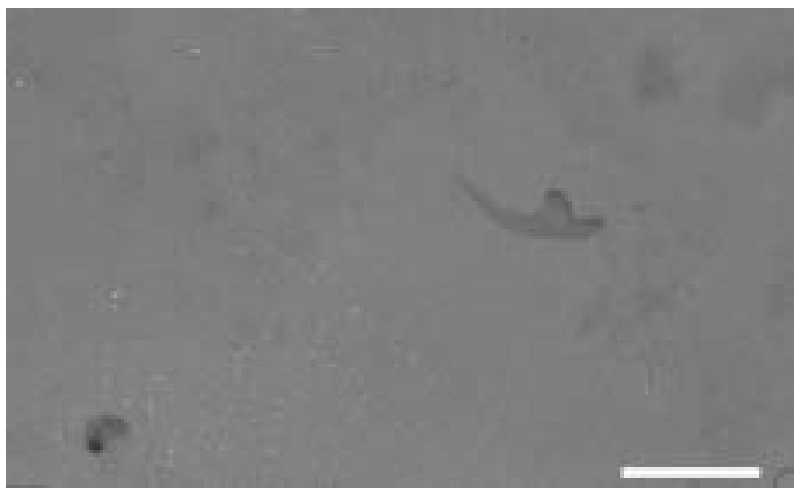

Fig. 3.-Fine-needle aspiration biopsy (haematoxylin and eosin staining, scale bar $=25 \mu \mathrm{m})$.

\section{BEFORE TURNING THE PAGE, INTERPRET THE ROENTGENOGRAM, THE CT SECTION AND THE BIOPSY. SUGGEST A DIAGNOSIS AND TREATMENT.}

\footnotetext{
*Dept of Radiology, ${ }^{\#}$ Dept of Chest Disease, Faculty of Medicine, Atatürk University, Erzurum, Turkey.

Correspondence: S. Suma, Dept of Radiology, Faculty of Medicine, Atatürk University, Erzurum, Turkey. Fax: 904423166340.
} 


\section{Interpretation}

Spherical and oval, well-marginated, homogeneous multiple radio-opacities were observed (fig. 1). The largest lesion was $4 \mathrm{~cm}$ in diameter. Quantitative evaluation of the lesions revealed $>60$ lesions. The lesions were located especially in the middle and lower zones. Spherical, round, well-marginated, homogeneous multiple nodular lesions were demonstrated on narrow window width (fig. 2). The lesions had a CT number equal to water (14 Hounsfield units). On wide window, lesions were widespread throughout the lung parenchymas. On lower sections in the right hemithorax, three or more lesions were demonstrated as cavitary lesions containing air. Figure 3 shows a shark-tooth shaped hooklet in the proteinaceous cyst. This finding was interpreted as Echinococcus granulosis. Immunological studies as well as Casoni and Weinberg tests confirmed this.

\section{Diagnosis: "Hydatid disease of the lung"}

\section{Treatment and clinical course}

As the lesions were widespread and multiple, medical therapy with mebandazole $\left(50 \mathrm{mg} \cdot \mathrm{kg}^{-1} \cdot \mathrm{day}^{-1}\right.$ for 6 months) was applied. To evaluate the response of the cysts to the therapy, the authors repeated the examinations at monthly intervals. Six months later, multiple, thin-walled cavitary lesions containing air in the lungs were observed on chest radiography (fig. 4). The size of the lesions decreased as well. On thorax $\mathrm{CT}$, the lesions were hardly visible on narrow window width, but on wide window level they were seen as thin-walled cavitary lesions containing air (fig. 5). All of the patient's symptoms disappeared during the eighth month. Eosinophilic count in blood became slightly elevated at the end of the seventh month.

\section{Discussion}

Hydatid disease is a parasitic infestation caused by the larval tapeworms E. granulosus and less frequently by E. multilocularis [1]. This disease is relatively common in the eastern part of Turkey. In that region, most of the people earn their living by raising cattle. Humans become accidentally infected, either through direct contact with infected dogs or by ingesting food, water, or soil containing the eggs of Echinococcus. Occasionally, the infection may be transmitted through the consumption of uncooked foods, such as salads. Nutrition with contaminated meat constitutes an important way of transmission of the disease to the humans. The liver $(75 \%)$ and lungs $(15 \%)$ are the organs most frequently involved in patients with hydatid disease caused by the larval stage of E. granulosus [2-4]. Cysts may be solitary or multiple. Multiple cysts are seen in about one-third of patients and are bilateral in $20 \%$; sometimes $>10$ cysts are seen [1].

E. granulosus infestation is a dynamic entity with different appearances depending on the degree and

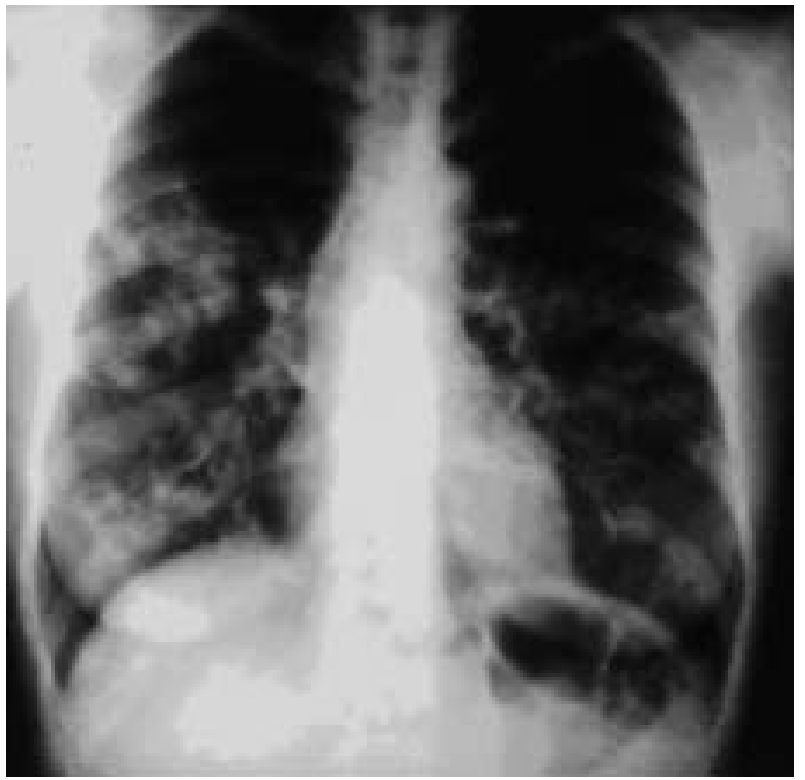

Fig. 4.-Posteroanterior chest radiography taken after medical therapy showing multiple, thin-walled cavitary images in the middle and lower zones.

severity of degenerative changes of the parasite [3]. Cysts are usually asymptomatic, and found incidentally on chest radiography [1]. Rupture of the cyst can result in sudden cough, haemoptysis and expectoration of cyst fluid, membranes and scolices $[1,4]$.

On plain chest radiography and thorax CT, one or more well-defined spherical or oval lesions of homogeneous density can be seen. CT numbers of the lesions are equal to that of water. The daughter cysts, when present, appear as curved septations [5].

Most pulmonary metastases have their origin in malignant tumours of the genitourinary and gastrointestinal tumours. Seminoma, Ewing's sarcoma, Wilms' tumour, osteogenic sarcoma, transitional cell carcinoma, teratocarcinoma, breast carcinoma and choriocarcinoma can be observed as multiple, oval, round, well-defined homogenous densities, especially on plain chest radiography. The demonstration of hypodense lesions with CT numbers equal to water on

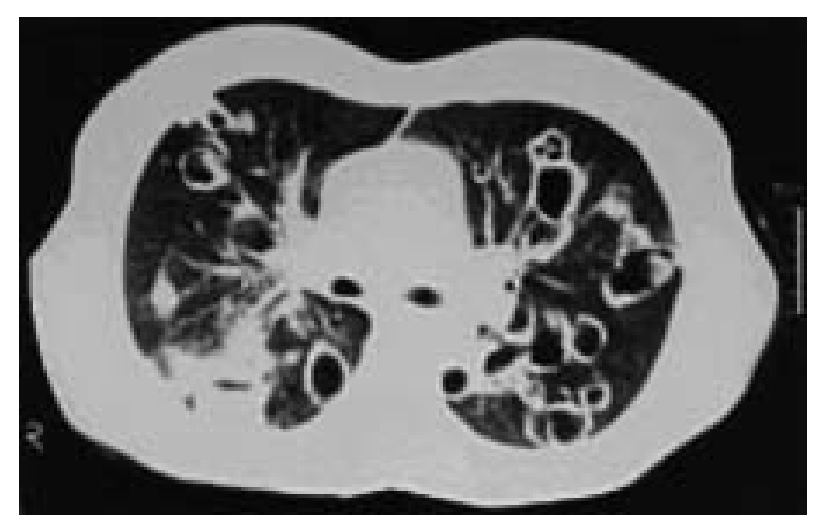

Fig. 5.-Axial computed tomography section with wide window level. Multiple, thin-walled cavitary images containing air can be seen. 
thorax CT and the anechoic cystic nature of the lesion on ultrasound (US) can provide a differential diagnosis of the hydatid disease from pulmonary metastatic lesions.

Surgery is widely accepted as the first choice in the treatment of the hydatid cyst, but the surgical treatment is sometimes difficult as in the present case. In these cases, medical therapy is advised [6, 7]. Changes observed during the medical therapy are generally examined in liver cyst-hydatid disease on US. The cyst size, intracystic sonographic pattern and the nature of the wall changes during therapy are reported in the literature [6].

In the present case, a decrease in the size of the lesions was detected. The cyst wall became thicker and the fluid content of the lesions was replaced by air. Antiparasitic drugs caused the detachment of the membranes and cell autolysis [6]. The air content of the cysts can be attributed to this detachment and autolysis. As a result of autolysis, the cyst wall comes into contact with the bronchial tree and air trapment occurs. This can be seen during the course of the disease without any therapy [7].

Hydatid disease should be taken into account when multiple radio-opacities are observed in the lungs on plain chest radiography, especially in the areas where this disease is endemic.

\section{References}

1. Beggs I. The radiology of hydatid disease. AJR Am J Roentganol 1985; 145: 639-648.

2. Beggs I. The radiological appearance of the hydatid disease of the liver. Clin Radiol 1983; 34: 277-283.

3. Hassen AG, Wahid H, Michel WB, Kathelyn D. Ultrasound examination of the hydatid liver. Radiology 1981; 139: 459-463.

4. David BL, Scott JM. Rupture of Echinococcal cysts: diagnosis, classification and clinical implications. $A J R$ Am J Roentganol 1986; 146: 391-394.

5. Saksouk FA, Fahl MH, Rizk GK. Computed tomography of pulmonary hydatid disease. J Comput Assist Tomogr 1986; 10: 226-232.

6. Bezzi M, Teggi A, De Rosa F, et al. Abdominal hydatid disease: US findings during medical treatment. Radiology 1987; 162: 91-95.

7. Godwin JD, Webb WR, Savoca CJ, Gamsu G, Goodman PC. Multiple, thin-walled cystic lesions of the lung. AJR Am J Roentganol 1980; 135: 593-604. 